Justin Kyale KoY ${ }^{1}$ David Andrew WARDELL ${ }^{2}$ Jean-Fiston MIKWA ${ }^{3}$ Joël Masimo KABUANGA Alphonse Maindo Monga NGongA Johan OszWALD 4

Charles Doumenge ${ }^{5,6}$

${ }^{1}$ Université de Kisangani

Faculté de sciences sociales,

administratives et politiques

Département des sciences politiques

et administratives

BP 2012, Kisangani

République démocratique du Congo

\section{${ }^{2}$ CIFOR}

Campus international de Baillarguet

TA C-105/D

34398 Montpellier cedex 5

France

${ }^{3}$ Université de Kisangani

Faculté de gestion des ressources naturelles renouvelables

Département des eaux et forêts

BP 2012, Kisangani

République démocratique du Congo

${ }^{4}$ Université Rennes 2

UMR LETG (UMR CNRS 5654)

Laboratoire Costel

Laboratoire Ecodiv (EA 1293, Rouen)

5, place Henri Le Moal

35000 Rennes

France

${ }^{5}$ Cirad

Unité de recherche Forêts et Sociétés

Université de Montpellier

Campus international de Baillarguet

TA C-105/D

34398 Montpellier cedex 5

France

${ }^{6}$ Forêts et Sociétés

Univ Montpellier

Cirad

Montpellier

France

Auteur correspondant /

Corresponding author:

Justin Kyale Koy - koykyale@yahoo.fr

\title{
Dynamique de la déforestation dans la Réserve de biosphère de Yangambi (République démocratique du Congo): variabilité spatiale et temporelle au cours des 30 dernières années
}

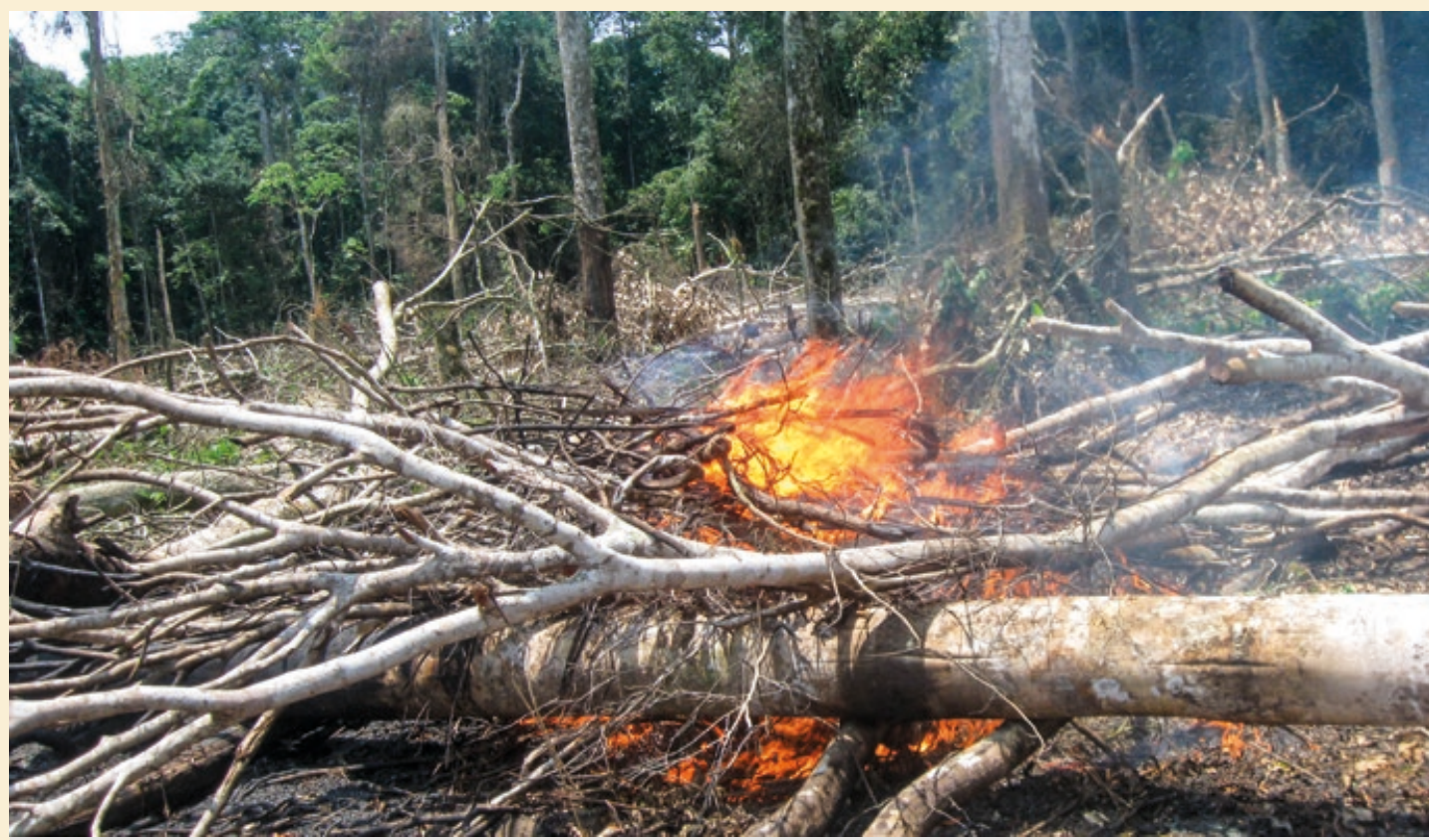

Photo 1.

Champ vivrier ouvert par les résidents du campement Beonga. Photo J. Kyale Koy.

Doi : 10.19182/bft2019.341.a31752 - Droit d'auteur (c) 2019, Bois et Forêts des Tropiques - (c) Cirad - Date de soumission : 18 mai 2018 ;

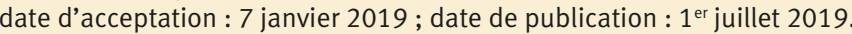

\section{Ocirad (a)}

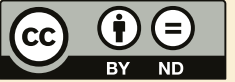

Licence Creative Commons :

Attribution - Pas de Modification 4.0 International.

Attribution-NoDerivatives 4.0 International (CC BY-ND 4.0)
Citer l'article / To cite the article

Kyale Koy J., Wardell D. A., Mikwa J.-F., Kabuanga J. M., Monga Ngonga A. M., Oszwald J., Doumenge C., 2019. Dynamique de la déforestation dans la Réserve de biosphère de Yangambi (République démocratique du Congo) : variabilité spatiale et temporelle au cours des 30 dernières années. Bois et Forêts des Tropiques, 341 : 15-28. Doi : https://doi.org/10.19182/ bft2019.341.a31752 


\section{RÉSUMÉ}

\author{
Dynamique de la déforestation dans \\ la Réserve de biosphère de Yangambi \\ (République démocratique du Congo) : \\ variabilité spatiale et temporelle \\ au cours des 30 dernières années
}

D’une superficie de 225000 ha, la Réserve de biosphère de Yangambi (République démocratique du Congo) est l'une des principales aires protégées menacées par l'anthropisation dans la région. Malgré son appartenance au réseau mondial des réserves de biosphère depuis les années 1970, les connaissances sur les dynamiques d'occupation du sol y sont encore lacunaires. Une démarche fondée sur l'analyse d'images (Landsat TM, $E T M+$ et OLI) et la réalité du terrain a été mise en œuvre pour étudier la dynamique d'occupation des sols de la réserve entre 1986 et 2016 . Le taux annuel de déforestation est passé de 0,18 \% au cours de la période $1986-2003$ à $0,38 \%$ en 2003 2016. Durant cette dernière période, la forêt primaire a connu un taux de déforestation plus élevé qu'en forêt secondaire (respectivement 4,5\% et 0,4\%). Quatre zones aux dynamiques socio-environnementales spécifiques ont été identifiées, nécessitant des approches de gestion différentes. Il s'agit notamment de la concession de l'INERA, des zones proches d'axes principaux de circulation, des zones situées sur des axes routiers plus ou moins abandonnés et des zones isolées du cœur de la réserve. Les principales causes de déforestation mentionnées par les habitants sont l'agriculture itinérante sur brûlis (54\% des ménages), l'exploitation artisanale des minerais (17\%) et la fabrication des pirogues (8\%). La pression démographique, sans amélioration du niveau de vie des communautés ou des techniques agricoles, et le développement du réseau routier, favorisant les échanges commerciaux, en sont les causes sous-jacentes. Cette dynamique de déforestation dans une forêt rattachée au réseau mondial des réserves de biosphère entame l'objectif fixé par l'État d'étendre le réseau d'aires protégées à $15 \%$ du territoire national.

\footnotetext{
Mots-clés : variabilité spatiale et temporelle, activités humaines, forêts denses, déforestation, réserve de biosphère, République démocratique du Congo.
}

\section{ABSTRACT}

\section{Dynamics of deforestation in the Yangambi Biosphere Reserve (Democratic Republic of Congo): spatial and temporal variability in the last 30 years}

The 225,000 ha Yangambi Biosphere Reserve (Democratic Republic of Congo) is one of the main protected areas under threat from human activities in the region. Despite its inclusion in the worldwide network of biosphere reserves since the 1970 s, there are still a great many gaps in knowledge as regards land use dynamics. An approach based on satellite image analysis (Landsat TM, ETM+ and OLI) and field studies was implemented to study the dynamics of land use in the reserve from 1986 to 2016. The annual rate of deforestation increased from $0.18 \%$ in $1986-2003$ to $0.38 \%$ from 2003 to 2016 . During the latter period, the deforestation rate was higher in primary forests $(4.5 \%)$ than secondary forests $(0.4 \%)$. Four zones with distinct socio-environmental dynamics were identified, each requiring a different management approach. These are the INERA logging concession, zones close to main roads, zones along more or less abandoned roads and isolated zones in the central part of the reserve. The main causes of deforestation cited by the inhabitants were slash-and-burn farming ( $54 \%$ of households), artisanal ore extraction (17\%) and the manufacture of dug-out canoes (8\%). The underlying causes are demographic pressure with few improvements in living conditions or agricultural techniques, and expansion of the road network, which promotes trade. These deforestation dynamics in a forest included in the worldwide network of biosphere reserves is compromising the State's goal of extending the network of protected areas to cover $15 \%$ of the country.

Keywords: Spatial and temporal variability, human activities, dense forests, deforestation, biosphere reserve, Democratic Republic of Congo.

\section{Dinámica de la deforestación en la Reserva de la Biosfera de Yangambi (República Democrática del Congo): variabilidad espacial y temporal en el curso de los 30 últimos años}

Con una superficie de 225000 ha, la Reserva de la Biosfera de Yangambi (República Democrática del Congo) es una de las principales zonas protegidas amenazadas por la antropización en la región. A pesar de su pertenencia a la red mundial de las reservas de la biosfera desde los años 1970, todavía hay lagunas en los conocimientos sobre las dinámicas de ocupación del suelo. Se aplicó un método basado en el análisis de imágenes (Landsat TM, $\mathrm{ETM}+$ y OLI) y la realidad del terreno para estudiar la dinámica de ocupación de los suelos de la reserva entre 1986 y 2016. La tasa anual de deforestación pasó del 0,18 \% durante el período 1986-2003 al 0,38\% en 2003-2016. Durante este último período, el bosque primario sufrió una tasa de deforestación más elevada que el bosque secundario (respectivamente 4,5\% y $0,4 \%)$. Se pudieron identificar cuatro zonas de dinámicas socioambientales específicas, lo que requirió enfoques de gestión diferentes. Se trata principalmente de la concesión del INERA, de las zonas próximas a los ejes principales de circulación, de las zonas situadas en ejes viales más o menos abandonados y en zonas aisladas del centro de la reserva. Las principales causas de deforestación mencionadas por los habitantes son la agricultura itinerante sobre chamicera (54\% de los hogares), la explotación artesanal de los minerales $(17 \%)$ y la fabricación de piraguas (8\%). La presión demográfica, sin mejora del nivel de vida de las comunidades ni de las técnicas agrícolas, y el desarrollo de la red de carreteras, que favorece los intercambios comerciales, son las causas subyacentes. Esta dinámica de deforestación en un bosque perteneciente a la red mundial de reservas de la biosfera inicia el objetivo fijado por el Estado de extender la red de zonas protegidas al $15 \%$ del territorio nacional.

Palabras clave: variabilidad espacial y temporal, actividades humanas, boscaje, deforestación, reserva de la biosfera, República Democrática del Congo. 


\section{Introduction}

Les forêts denses de la République démocratique du Congo (RDC) occupent près de 115 millions d'hectares, soit plus de $60 \%$ des forêts denses d'Afrique centrale (Ernst et al., 2012). Elles subissent diverses pressions anthropiques qui en réduisent chaque année la superficie (Potapov et al., 2012). Le taux de déforestation annuelle en RDC varie de 0,18 à $0,46 \%$ en fonction des méthodes utilisées, des sites étudiés, des types de végétation suivis ou des périodes considérées (Duveiller et al., 2008 ; De Wasseige et al., 2012 ; Molinario et al., 2015 ; Muyaya Kalambay et al., 2016). Si le taux moyen national de 0,22 \% reste relativement faible par rapport à la moyenne mondiale, il reste cependant le plus élevé des pays du bassin du Congo (De Wasseige et al., 2012).

Le souhait du gouvernement de classer au moins $15 \%$ de la superficie du territoire national en aires protégées traduit l'une des stratégies de protection des milieux naturels, en particulier forestiers. Toutefois, l'administration peine à mettre cette politique en œuvre (Pélissier et al., 2015) car ces aires protégées sont souvent menacées par des activités anthropiques (Potapov et al., 2012). En Afrique centrale, particulièrement en RDC, la quasi-inexistence des politiques d'affectation des terres favorise la déforestation et la dégradation des forêts. L'agriculture itinérante sur brûlis est la principale cause de la déforestation et de la dégradation, suivie par l'expansion des infrastructures et le développement du secteur minier ou du bois (Gillet et al., 2016). Toutefois, des études de cas et des analyses précises des dynamiques locales manquent encore pour éclairer les processus en cause et modéliser les trajectoires futures des socio-écosystèmes face aux changements globaux.

La déforestation ou la dégradation des forêts sont généralement évaluées à l'aide d'outils de télédétection à haute résolution spatiale (Molinario et al., 2015), voire, plus localement, à l'aide d'imageries à très haute résolution (Semeki Ngabinzeke et al., 2016). L'acquisition de ces données nécessite l'engagement de coûts financiers élevés (Willis, 2015). Dans le contexte actuel de la RDC, le recours aux images Landsat pour étudier l'évolution des paysages est la solution la plus économique et la plus pratique (Oszwald et al., 2011).

Comme pour la plupart des aires protégées de la RDC, la Réserve de biosphère de Yangambi (RBY) accuse un déficit d'études relatives au changement du couvert végétal et aux facteurs de déforestation et de dégradation forestière. En dépit de son rattachement au réseau mondial des réserves de biosphère depuis 1977, aucun suivi de dynamique de la végétation n'y a été entrepris. Pourtant, sa situation, dans la cuvette centrale, en bordure du fleuve Congo et en périphérie d'un des plus grands centres urbains du pays (Kisangani), en fait un modèle d'aire protégée particulièrement intéressant à étudier dans un contexte de tensions entre la conservation de la biodiversité et le développement économique, surtout dans les zones d'influence de grandes villes (Walters et al., 2016).

La présente étude visait à combler ce déficit en contribuant à une meilleure connaissance de la dynamique d'occupation des sols dans les forêts classées en RDC et des facteurs qui l'orientent. Elle permet de mieux informer les politiques publiques d'aménagement, de protection et d'utilisation durable des forêts de la cuvette centrale congolaise en identifiant les principaux moteurs du changement et les zones fortement anthropisées dans et en périphérie des aires protégées. L'étude s'est attachée plus spécifiquement à vérifier les deux hypothèses suivantes: les activités anthropiques et la déforestation varient dans l'espace et au cours du temps dans la RBY ; les principaux facteurs de déforestation sont les types d'activités humaines, la démographie et la proximité aux voies de communication, d'autres facteurs pouvant aussi s'avérer primordiaux en fonction des situations locales.

Au regard de ces hypothèses, l'objectif poursuivi dans cet article était d'analyser la dynamique de la déforestation dans la RBY en vue d'en cerner la variabilité spatiale et temporelle au cours des 30 dernières années et les facteurs qui l'ont orientée. L'atteinte de cet objectif peut permettre de parfaire le plan d'aménagement de la RBY et de proposer des règles de gestion et de gouvernance mieux adaptées aux dynamiques en cours.

\section{Matériels et méthodes}

\section{La Réserve de biosphère de Yangambi}

La RBY a été créée par l'Ordonnance n 121 Agri, du 25 novembre 1939 (INEAC, 1939). Elle est localisée au nord-est de la RDC, dans la province de la Tshopo. Sa plus proche limite se situe à environ $60 \mathrm{~km}$ au nord-ouest de la ville de Kisangani, capitale provinciale. Elle couvre une superficie légale évaluée à environ 225000 ha (INEAC, 1939). La présente étude prend en compte la réserve et sa proche périphérie, couvrant 273955 ha et incluant la réserve de N'Gazi et la concession de l'INERA (Institut national pour l'étude et la recherche agronomiques), deux domaines annexés depuis sa reconnaissance en 1977 par l'Unesco (figure 1).

La réserve occupe une zone de plateaux dont l'altitude varie entre 400 et $500 \mathrm{~m}$. Elle bénéficie d'un climat équatorial, chaud et humide et à saisons sèches peu marquées (Beguin, 1958). Les précipitations annuelles varient entre 1600 et $2200 \mathrm{~mm}$, avec une température moyenne annuelle supérieure à $18{ }^{\circ} \mathrm{C}$ (Mohymont et Demarée, 2006). En avril (mois le plus chaud), la température moyenne est de $25,8^{\circ} \mathrm{C}$, alors qu'en juillet (mois le plus froid) elle est de $24,2{ }^{\circ} \mathrm{C}$ (Kombele, 2004). La réserve est bordée au sud-ouest par le fleuve Congo; elle est sillonnée par les affluents de ce dernier dont les principaux sont les rivières Aruwimi (au nord) et Lindi (à l'est).

Les études antérieures ont permis d'identifier 128 essences forestières susceptibles de fournir du bois d'œuvre (Donis, 1956), 239 espèces de poissons (Gosse, 1963) et 44 espèces de mammifères (Toirambe et al., 2011). Cependant, ces statistiques doivent être prises avec précaution car aucune étude approfondie n'a été menée pour la plupart des groupes taxonomiques, comme l'avaient déjà souligné Poll et Gosse (1963). 
La réserve et sa périphérie abritent l'agglomération de Yangambi ainsi que divers villages et campements répartis le long du fleuve et des axes routiers et à l'intérieur ou autour de ses limites (figure 2). Outre les maisons construites en matériaux durables à Yangambi-centre, les villages et campements riverains de la RBY sont caractérisés par un habitat construit en matériaux issus de la forêt. Les axes routiers qui traversent ou bordent la réserve permettent le déplacement de la population à l'intérieur de la région et vers les grandes agglomérations, notamment Kisangani, Yangambi, Isangi et Bengamisa (figure 2). Toutefois, certains ne sont actuellement praticables qu'à pied ou à deux-roues.

\section{Images satellitaires}

Les images de la série Landsat corrigées géométriquement (niveau L1b) ont été utilisées pour cartographier la dynamique de l'occupation du sol dans la RBY. Trois dates ont été retenues : 1986 (Thematic Mapper: TM), 2003 (Enhanced Thematic Mapper+ : ETM+) et 2016 (Operational Land Imager : OLI). Le choix des dates a été conduit en fonction de la disponibilité et de la qualité des données. De plus, nous avons essayé de faire coïncider ces dates avec des dates clés du développement de la région : la découverte des minerais de diamant, qui a entraîné l'installation de nombreux campements à l'intérieur et autour de la réserve à partir de l'année 1986 ; la fin de la deuxième guerre civile et le début de la prise en charge des écogardes de Man and Biophere (MAB) par l'État congolais en 2003 (dans le but de renforcer leur capacité de contrôle ou de surveillance) ; la période de concentration des interventions des projets à Yangambi entre 2010 et 2016 (WWF, UICN, CIFOR), nécessitant l'évaluation de leurs impacts sur la réduction des activités humaines.

En situation de forêt dense humide, la zone d'étude présente une forte nébulosité. De ce fait, seules les images acquises durant la période sèche ont été privilégiées pour l'étude (tableau I).

\section{Prétraitements des images}

Figure 1.

Domaines constitutifs de la Réserve de biosphère à Yangambi.

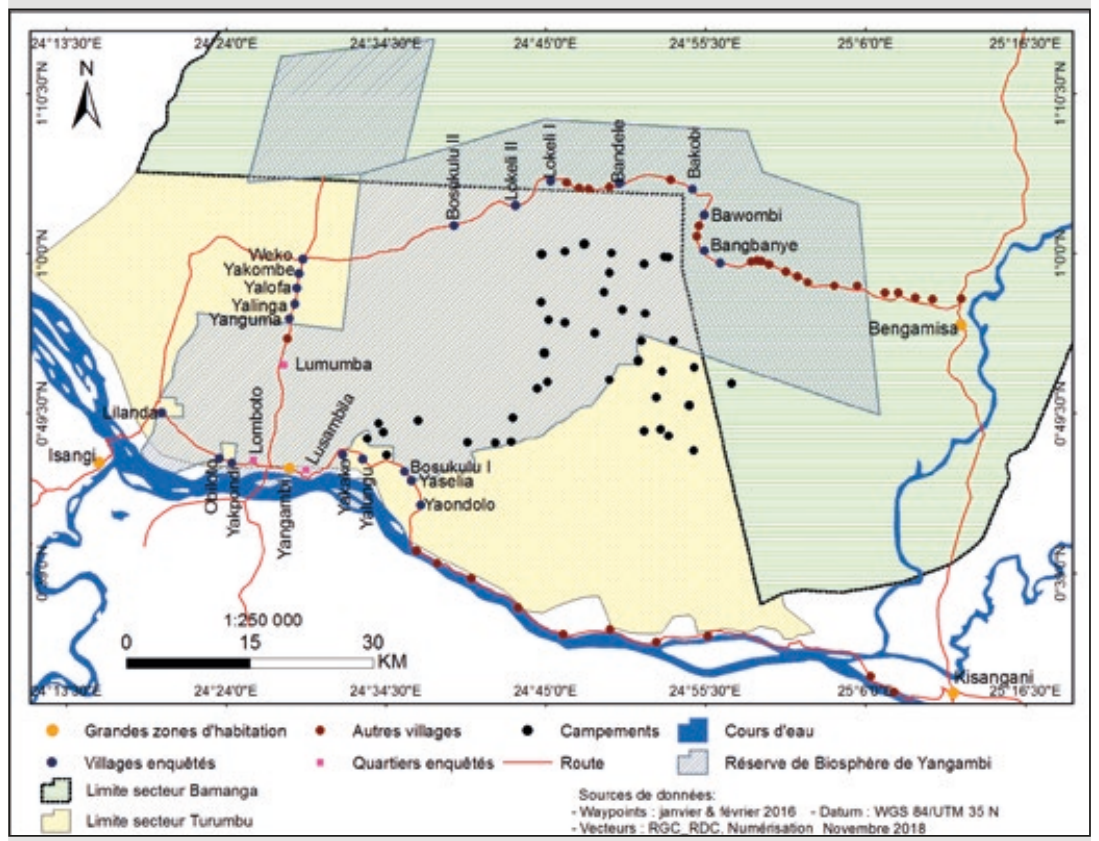

Figure 2.

Carte de situation de la Réserve de biosphère à Yangambi : les villages, les quartiers, les campements et la zone périphérique.
Les images ont d'abord été prétraitées avec des corrections géométriques, radiométriques et atmosphériques. Ensuite, elles ont été mosaïquées et masquées afin de créer les premières compositions colorées. La zone d'étude est répartie en trois scènes Landsat (path and row : 176/059, 176/060 et 177/059). Les images de la ligne (path) 176 ont été géoréféncées dans le système des coordonnées WGS 84/UTM 35 Nord. Celles de la ligne 177 avaient un système des coordonnées différent (WGS 84/ UTM 34 Nord). Une reprojection a été réalisée sur toutes les images de la ligne 177 pour uniformiser le système des coordonnées et faciliter la superposition des images. L'orthorectification de l'image de 2016 a été réalisée avec les coordonnées géographiques (points de contrôle) réparties sur la zone d'étude. II s'agit entre autres des carrefours des routes (situés à Yambelo, Yangambi, Lilanda et Weko), des ponts fluviaux (Lilanda, Boonde, Lobilo et Lubuye) et des crêtes des îles présentes sur le fleuve Congo. Les autres images (1986 et 2003) ont été orthorectifiées en référence à celle de 2016. La précision géométrique du calage entre chacune des images antérieures et celle de 2016 obtenue étant inférieure à 1 pixel, l'analyse des changements est efficiente (Mas, 2000). 
Tableau I.

Caractéristiques des images satellitaires utilisées.

\begin{tabular}{|c|c|c|c|c|}
\hline Capteur & Path/row & $\begin{array}{l}\text { Date } \\
\text { d'acquisition }\end{array}$ & $\begin{array}{l}\text { Bande } \\
\text { spectrale }(\mu \mathrm{m})\end{array}$ & $\begin{array}{l}\text { Résolution } \\
\text { spatiale (m) }\end{array}$ \\
\hline \multirow[t]{11}{*}{ Operational Land Imager (OLI) } & $176 / 059$ & $05 / 04 / 2016$ & $0,433-0,453$ & 30 \\
\hline & $176 / 060$ & $05 / 04 / 2016$ & $0,450-0,515$ & 30 \\
\hline & $177 / 059$ & $07 / 01 / 2016$ & $0,525-0,600$ & 30 \\
\hline & & & $0,630-0,680$ & 30 \\
\hline & & & $0,845-0,885$ & 30 \\
\hline & & & $1,560-1,660$ & 30 \\
\hline & & & $2,100-2,300$ & 30 \\
\hline & & & $0,500-0,680$ & 15 \\
\hline & & & $1,360-1,390$ & 30 \\
\hline & & & $10,30-11,30$ & 100 \\
\hline & & & $11,50-12,50$ & 100 \\
\hline \multirow[t]{8}{*}{ Enhanced Thematic Mapper+ (ETM+) } & $176 / 059$ & $03 / 01 / 2003$ & 0,45-0,52 (bleu) & 30 \\
\hline & $176 / 060$ & $19 / 12 / 2002$ & $0,52-0,60$ (vert) & 30 \\
\hline & $177 / 059$ & $26 / 12 / 2002$ & 0,63-0,69 (rouge) & 30 \\
\hline & & & 0,761-0,90 (PIR) & 30 \\
\hline & & & 1,55-1,75 (MIR) & 30 \\
\hline & & & $10,40-12,50$ (IRT) & 60 \\
\hline & & & $2,08-2,35$ (MIR) & 30 \\
\hline & & & 0,5-0,9 (PANCHRO) & 15 \\
\hline \multirow[t]{7}{*}{ Thematic Mapper (TM) } & $176 / 059$ & $14 / 02 / 1986$ & 0,45-0,52 (Bleu) & 30 \\
\hline & $176 / 060$ & $14 / 02 / 1986$ & $0,52-0,60$ (Vert) & 30 \\
\hline & $177 / 059$ & $16 / 01 / 1986$ & 0,63-0,69 (rouge) & 30 \\
\hline & & & 0,76-0,90 (PIR) & 30 \\
\hline & & & 1,55-1,75 (MIR) & 30 \\
\hline & & & $10,40-12,50($ IRT) & 120 \\
\hline & & & 2,08-2,35 (MIR) & 30 \\
\hline
\end{tabular}

Les corrections atmosphériques ont été réalisées à partir du module FLAASH (Fast Line-of-sight Atmospheric Analysis Hypercubes) basé sur le modèle atmosphérique MODTRAN (MODerate resolution atmospheric TRANsmission) (Arabi et al., 2016 ; Rosas et al., 2017). En premier lieu, un étalonnage a été réalisé pour définir le type de satellite, la date d'acquisition de l'image et l'angle d'élévation solaire. Ensuite, le calcul de la radiance a été réalisé. Puis, les unités physiques des bandes ont été ajustées à l'aide d'un facteur de correction 10. Cet ajustement a été réalisé car la radiance est donnée en $\mathrm{W} / \mathrm{m}^{2} / \mathrm{sr} / \mathrm{nm}$, tandis que FLAASH demande les données en $\mu \mathrm{W} / \mathrm{cm}^{2} / \mathrm{sr} / \mathrm{nm}$.

Par ailleurs, les images ont été préparées pour la correction atmosphérique en changeant l'interleave. Cette conversion a conduit à la création des images de radiance correctement configurées pour le format d'entrée de FLAASH. Les images acquises, au format BSQ (qui est généralement le format de fichier par défaut), ont été converties en format de fichier BIP pour le traitement spectral.
Les mosaïques ont été réalisées en veillant à éliminer les nuages et les voiles, autant que possible, en retenant les portions d'images de meilleure qualité. La création des masques a été assurée à l'aide d'une couche vectorielle reprenant la Réserve de biosphère de Yangambi selon les limites de l'INEAC (Toirambe et al., 2011).

Pour une meilleure discrimination de la végétation, les compositions colorées ont été visualisées en composition fausses couleurs (rouge, proche infrarouge et infrarouge respectivement pour les canaux rouge, vert et bleu). Pour les mosaïques issues des capteurs TM (1986) et ETM+ (2003), les bandes 3, 4 et 5 ont été utilisées. Par ailleurs, la mosaïque de 2016, issue des images OLI, a nécessité l'utilisation des bandes 4, 5 et 6 pour visualiser la composition colorée en couleur naturelle. Le logiciel ENVI 4.7 a été utilisé pour les prétraitements des images et la classification de l'occupation du sol. 


\section{Sélection des zones d'entraînement}

Les coordonnées géographiques correspondant à chaque classe d'occupation du sol ont été relevées à l'aide d'un GPS de type Garmin MAP 62stc entre janvier et février 2016. Chaque coordonnée représente les éléments constitutifs d'une classe. Afin d'obtenir une meilleure représentativité des diverses classes forestières, le prélèvement des points a été réalisé à la fois dans le cœur de la réserve et dans les zones externes à proximité des villages. Chaque point fait l'objet d'une description du milieu de référence. Au total, 842 points de mesure ont été prélevés. Cet ensemble a été scindé en deux groupes de données : 547 ayant servi à la classification de l'occupation du sol en 2016 (soit 64,96\% de coordonnées géographiques) et 295 points ayant servi à la validation de la classification de 2016.

\section{Classes d'occupation du sol}

L'occupation du sol a été définie en cinq classes principales. La première classe est la forêt dite « primaire » (FP). Elle comprend les forêts denses humides de terre ferme, sempervirentes ou semi-caducifoliées, et les forêts marécageuses et inondables. La composition floristique de ces forêts est caractérisée par les espèces telles que Scorodophloeus zenkeri, Cynometra hankei, Prioria oxyphylla, Prioria balsamifera, Pericopsis elata, Brachystegia laurentii, Gilbertiodendron dewevrei (Van Wambeke et Evrard, 1954).

La deuxième classe correspond à la forêt dite « secondaire » (FS) formée d'un complexe de stades de transition entre les formations récentes de reconstitution du couvert forestier après défrichement (jeune forêt secondaire) et la vieille forêt secondaire (Van Wambeke et Evrard, 1954). On y rencontre les espèces telles que Ricinodendron heudelotii, Pentaclethra macrophylla, Albizia adianthifolia, Myrianthus arboreus, Alstonia boonei, Petersianthus macrocarpus, Musanga cecropioides, Vernonia conferta, Macaranga spinosa, Harungana madagascariensis et Anthocleista schweinfurthii. Les stades jeunes présentent un sous-bois très dense, dominé par Oncoba welwitschii, Tabernaemontana crassa, Tabernaemontana penduliflora. Palisota ambigua, Alchornea floribunda et Haumania liebrechtsiana dominent quant à elles le sous-bois des stades adultes (Van Wambeke et Evrard, 1954). Les parcelles expérimentales sylvicoles installées à Yangambi avant 1960 sont également associées à cette classe.

Les " jachères et champs mis en culture » (ICC) forment la troisième classe d'occupation du sol. Ce sont des parcelles cultivées ou mises en repos pour être défrichées après quelques années selon un système rotatif.

La quatrième classe comprend les "sols nus et les espaces bâtis » (SNB). C'est une mosaïque constituée des zones bâties, des végétations herbacées et des jardins-vergers familiaux. Ces éléments paysagers sont localisés à proximité des villages ou le long des routes. Le réseau routier fait partie de cette classe.

Enfin, la dernière classe est celle rassemblant toutes les surfaces d'eau libre constituées des cours d'eau et des rivières qui parcourent la réserve.

\section{Classification des images satellites}

L'algorithme de classification selon le maximum de vraisemblance (Soungalo et al., 2015) a été appliqué à tous les pixels des images sur base des paramètres statistiques décrivant chaque signature spectrale obtenue (les moyennes et les écarts-types). Cette méthode a l'avantage de fournir à chaque pixel, en plus de la classe à laquelle il a été affecté, un indice de certitude lié à ce choix (Barima et al., 2009).

\section{Validation des cartes d'occupation du sol}

La validation par pixels a permis d'évaluer la qualité de la classification sur la zone d'étude. Les points ont été attribués aux classes correspondant à l'interprétation des pixels qui les entourent, avant leur superposition sur la meilleure classification de chaque classe (Thi-Thanh-Hiên Pham et Dong-Chen $\mathrm{He}, 2013)$. La création des matrices de confusion, validant la qualité de la classification, a permis de déterminer le nombre de pixels correctement classés à partir des données issues du terrain. Lesdites matrices indiquent une moyenne de $94,8 \%$, $93,6 \%$ et $93,2 \%$ des pixels correctement classés respectivement pour les années 1986, 2003 et 2016. La précision globale de classification a été mesurée par le coefficient Kappa (Soungalo et al., 2015). Ce dernier est de $95 \%, 96 \%$ et $96 \%$ respectivement pour les années 1986, 2003 et 2016.

Après la validation, les classes d'occupation du sol ont été compilées en valeur absolue (ha) et en pourcentage afin d'analyser les changements intervenus durant les périodes d'étude (Latendresse et al., 2008). La détection des changements entre les images a été obtenue grâce à la matrice de transition créée pour identifier les fréquences de transition entre les classes au cours de l'intervalle de temps étudié (Barima et al., 2009). Cette matrice est obtenue par le croisement des cartes d'occupation du sol de deux périodes comparées (1986-2003, 2003-2016), réalisé à l'aide du logiciel ENVI 4.7.

Le taux de déforestation (par période) dans la zone d'étude a été calculé en recourant à l'équation suivante (Caloz et Collet, 2001) :

T déforestation $(\%)=\frac{S 2-S 1}{S} \times 100$

où S2 est l'étendue occupée par la forêt (primaire ou secondaire) en année terminale de la période, S1 est l'étendue occupée par la forêt (primaire ou secondaire) en année initiale de la période, $\mathrm{S}$ est la superficie occupée par la forêt (primaire et secondaire) en année initiale.

Enfin, la mise en page cartographique a été réalisée à l'aide du logiciel ArcGIS 10.1.

\section{Entretiens avec les communautés}

Les entretiens semi-structurés et les focus group ont été réalisés de février à mai et en octobre 2015. Ils visaient à l'identification des sites et des activités responsables de la déforestation (campements, agriculture, exploitation minière et forestière). Un échantillon de 300 personnes a été interrogé dans 20 villages et trois quartiers de Yangambi-centre (voir figure 2 pour l'ensemble des villages). 


\section{Résultats}

\section{Dynamique d'occupation du sol}

Les trois cartes ci-après montrent clairement la diminution des surfaces forestières au cours des trente dernières années (figure 3). Le taux de déforestation est passé de 3,1\% du territoire cartographié entre 1986 et 2003 soit une perte de plus de 8100 ha des forêts primaire et secondaire - à 4,9\% entre 2003 et 2016 (soit la disparition supplémentaire de plus de 12600 ha de forêts ; tableau II). Cela se traduit par un doublement des taux de déforestation moyenne annuelle entre la première et la seconde période $(0,18 \%$ à $0,38 \%$ par an). La forêt primaire seule a diminué de plus de 3300 ha entre 1986 et 2003 et d'environ 11500 ha entre 2003 et 2016.

Au cours de la première période (1986-2003), la déforestation est localisée essentiellement à Yangambi-centre et dans le sud de la concession de l'INERA et, de manière plus ponctuelle, le long de l'axe routier Bengamisa-Weko ou vers Lilanda (axe Yangambi-Isangi ; figures 2 et 3). Ces tendances se sont renforcées au cours de la seconde période, avec une déforestation généralisée de la concession de l'INERA, une expansion importante dans la région de Bengamisa (est de la RBY) et des marges sud de la RBY (entre l'axe routier et le fleuve Congo). Ailleurs, elle apparaît principalement autour de petits campements forestiers ou du réseau hydrographique. S'étendant et se déplaçant au cours du temps, elle impacte plus fortement les forêts primaires au cours de la seconde période.

\section{Taux de changement entre les périodes étudiées}

La matrice de transition (tableau III) révèle que 97,8 \% des superficies occupées par la forêt primaire en 1986 n'ont pas connu de modifications en 2003. C'est essentiellement la forêt secondaire qui a subi l'impact de la déforestation (environ $20 \%$ de forêt secondaire a disparu en 2003), transformée surtout en jachères et champs cultivés (pour 14,2 \%) ou en sols nus et espaces bâtis (pour 5,4 \%).

Au cours de la période 2003-2016 (tableau IV), la forêt primaire a été plus fortement impactée que pendant la période précédente : un peu moins de $5 \%$ des espaces forestiers primaires ont changé d'état en 13 années (contre un peu plus de $2 \%$ au cours des 17 années de la période précédente). Elle a été transformée en jachères et champs cultivés ou en sols nus et espaces bâtis sur 3,5\% de sa surface, voire dégradée en forêt secondaire sur $1,1 \%$ de sa surface. Au contraire, près de $89 \%$ de la superficie occupée par la forêt secondaire s'est maintenue en l'état, un peu moins de $8 \%$ étant transformée en jachères et champs cultivés ou en sols nus et espaces bâtis.

\section{Activités humaines modifiant les forêts}

Les résultats des entretiens montrent que l'agriculture itinérante sur brûlis (photo 1) constitue la principale source de revenu pour $98 \%$ des ménages $(n=300)$. Environ $54 \%$ de ceux qui la pratiquent défrichent dans la

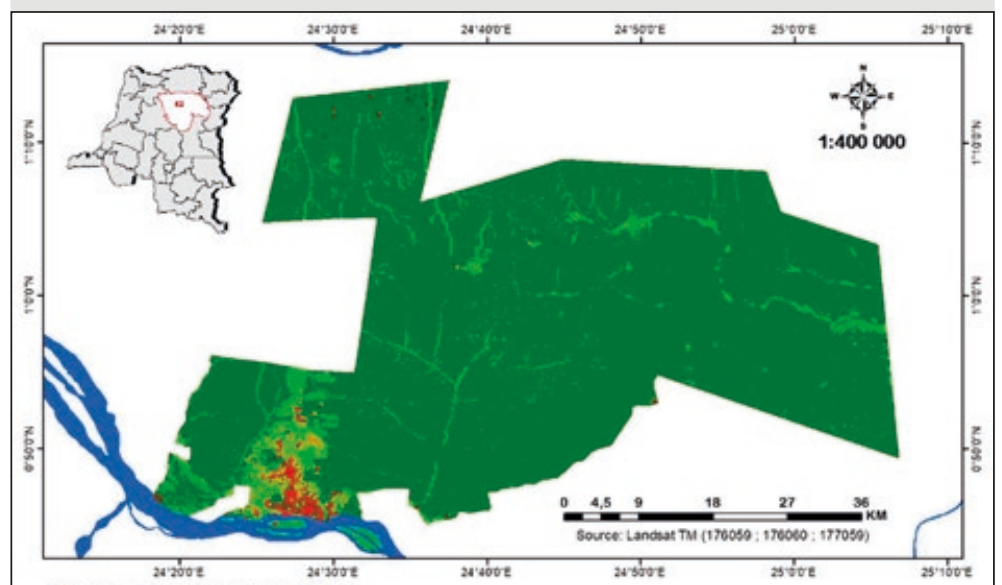

Occupation du sol en 1986

Forêt primaire 1 Forêt secondaire Jachere et champs Sol nu et batis Eau

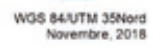

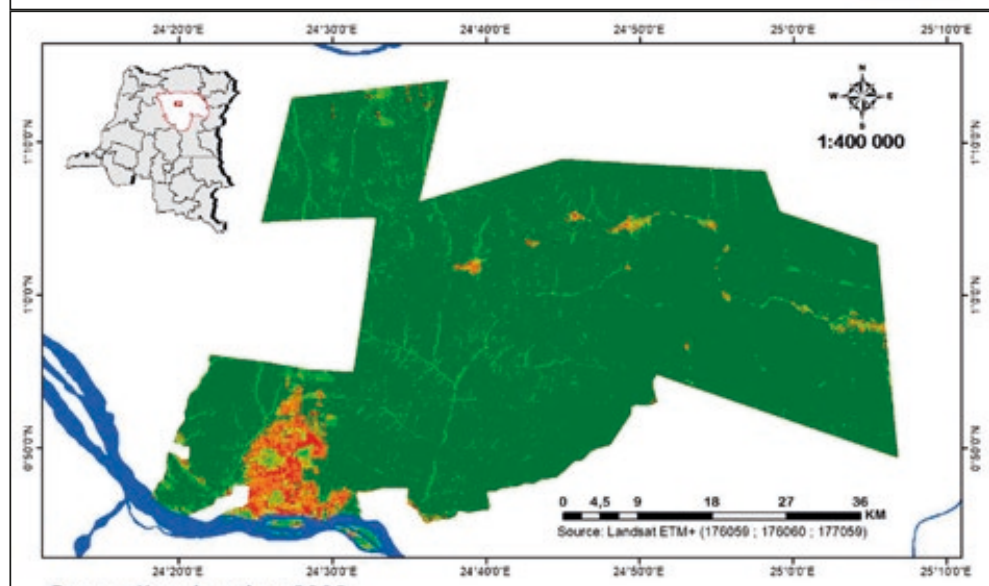
Occupation du sol en 2003

Forêt primaire 10 Forêt secondaire Wos BuVTM 3 SSNord
Novembre, 2018

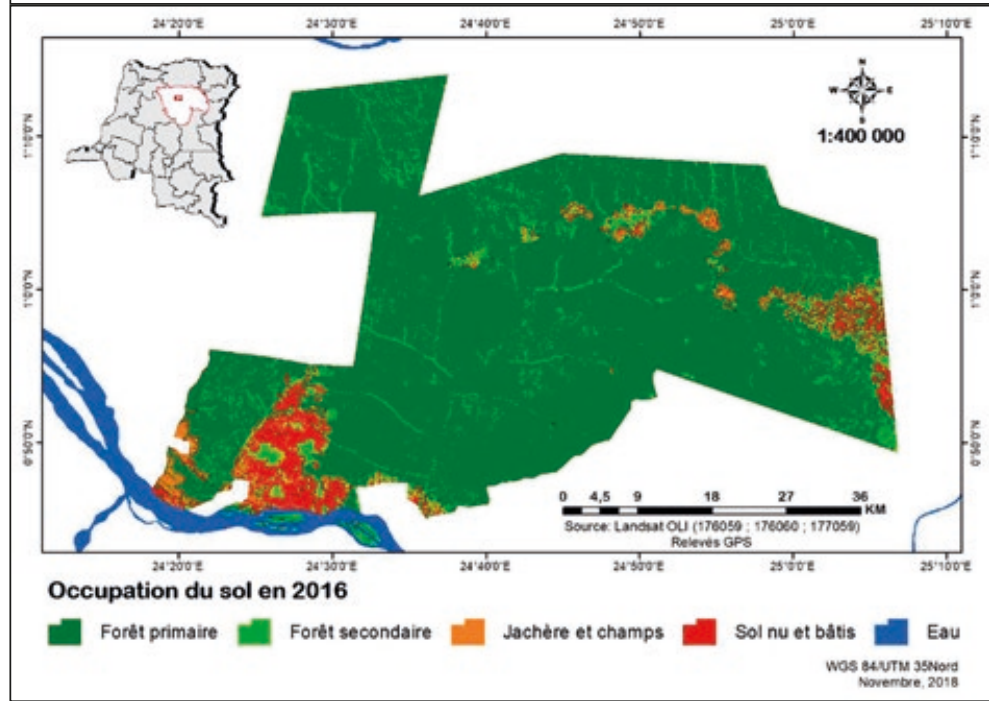

Figure 3.

Cartes de l'occupation du sol en 1986, 2003 et 2016

dans la zone d'étude. 
Tableau II.

Étendues et pertes du couvert forestier de la Réserve de biosphère de Yangambi entre 1986 et 2016.

\begin{tabular}{|c|c|c|c|c|c|c|c|}
\hline \multirow[t]{2}{*}{ Classes } & \multicolumn{3}{|c|}{ Superficie (ha) } & \multicolumn{2}{|c|}{ Bilan (ha) } & \multicolumn{2}{|c|}{ Taux de déforestation (\%) } \\
\hline & 1986 & 2003 & 2016 & $1986-2003$ & 2003-2016 & $1986-2003$ & 2003-2016 \\
\hline FP & 250480 & 247179 & 235633 & -3301 & -11547 & $-1,2$ & $-4,5$ \\
\hline FS & 15084 & 10248 & 9147 & -4836 & -1101 & $-1,8$ & $-0,4$ \\
\hline $\mathrm{JCC}$ & 1205 & 9217 & 11426 & 8012 & 2209 & & \\
\hline SNB & 2585 & 3097 & 13697 & 512 & 10600 & & \\
\hline Eau & 4101 & 4214 & 4052 & 113 & -162 & & \\
\hline Total & 273955 & 273955 & 273955 & & & $-3,1$ & $-4,9$ \\
\hline
\end{tabular}

FP : forêt primaire ; FS : forêt secondaire ; JCC : jachères et champs mis en culture ; SNB : sols nus et bâtis.

Tableau III.

Changements d'occupation des sols opérés entre 1986 et 2003 (en \%).

\begin{tabular}{|c|c|c|c|c|c|c|}
\hline \multirow{2}{*}{\multicolumn{2}{|c|}{ Classes }} & \multirow[b]{2}{*}{ FP } & \multirow[b]{2}{*}{ FS } & \multirow{2}{*}{$\begin{array}{c}1986 \\
\text { JCC }\end{array}$} & \multirow[b]{2}{*}{ SNB } & \multirow[b]{2}{*}{ Eau } \\
\hline & & & & & & \\
\hline \multirow[t]{7}{*}{2003} & FP & 97,8 & 2 & 0,2 & 0 & 0 \\
\hline & FS & 1 & 78 & 16,8 & 4,2 & 0 \\
\hline & JCC & 0,2 & 14,2 & 76 & 8,3 & 1,3 \\
\hline & SNB & 1 & 5,4 & 7 & 85,5 & 1,1 \\
\hline & Eau & 0 & 0,4 & 0 & 2 & 97,6 \\
\hline & Total de classes & 100 & 100 & 100 & 100 & 100 \\
\hline & Changement des classes & 2,2 & 22 & 24 & 14,5 & 2,4 \\
\hline
\end{tabular}

Tableau IV.

Changements d'occupation des sols opérés entre 2003 et 2016 (en \%).

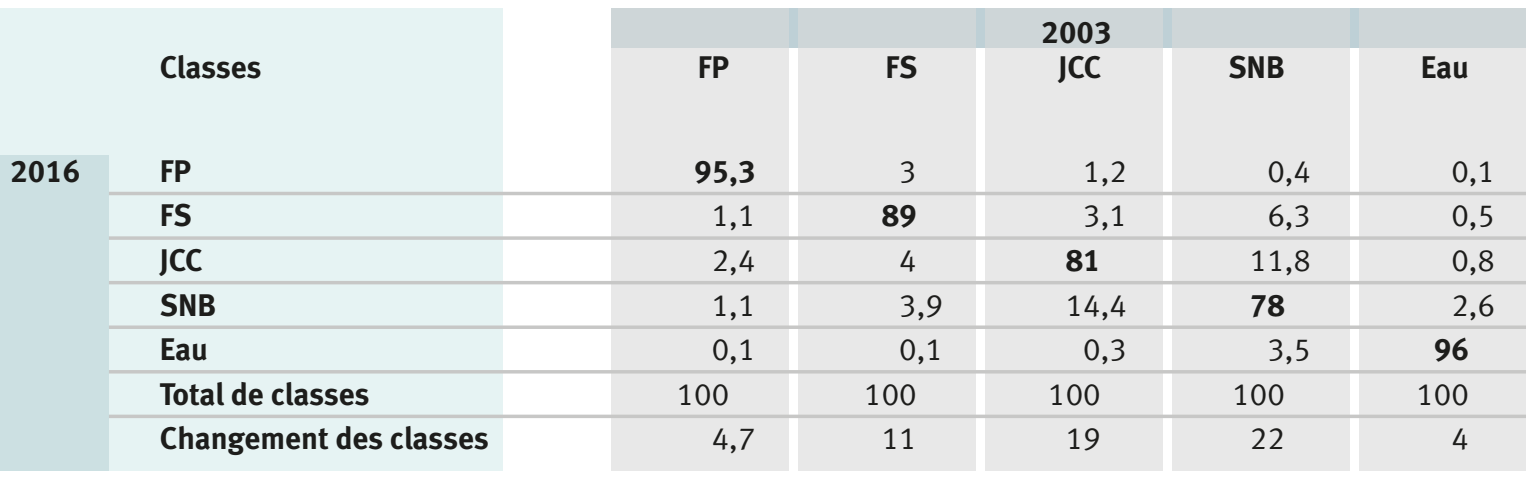

FP : forêt primaire ; FS : forêt secondaire ; JCC : jachères et champs mis en culture ; SNB : sols nus et bâtis. 
réserve (essentiellement ceux qui sont localisés sur l'axe Yambelo-Weko, à Yangambi-centre, vers Lilanda et dans les campements; figure 2). Les paysans cultivent dans la forêt primaire (11\%) pour acquérir le droit de propriété coutumière des forêts secondaires et jachères. Les jachères sont habituellement exploitées par $74 \%$ des ménages. La dimension moyenne des champs est d'environ 1 ha. Les paysans défrichent en moyenne un champ par an. La durée des jachères varie de 1 à 15 ans, avec une moyenne d'environ 3,5 ans. La distance moyenne des champs nouvellement ouverts par rapport au village varie de $500 \mathrm{~m}$ à $4 \mathrm{~km}$, avec une moyenne de $1,8 \mathrm{~km}$. Ainsi, les classes de forêt primaire et secondaire modifiées par cette activité sont celles localisées non loin des zones habitées.

La survie des communautés dépend également de l'exploitation artisanale d'or et de diamants (17\%) et de la construction des pirogues (8\%). La modification de la forêt (primaire) par l'activité minière commence par l'installation des campements (photo 2). L'exploitation proprement dite consiste à creuser des trous le long du lit de la rivière (photo 3) ou dans la forêt. Lorsqu'ils sont abandonnés, ces sites miniers ne sont pas remblayés, ni réhabilités. La reconstitution de l'humus étant difficile, le recrû forestier demeure lent.

La modification de la forêt (primaire) résulte de l'installation des campements par les constructeurs, l'agriculture (photo 5) et la coupe des arbres ainsi que la construction des pirogues (photo 4). C'est l'une des activités responsables de l'installation de nombreux campements par les fabricants qui sont à la recherche de Prioria balsamifera.

\section{Discussion}

Les dynamiques de déforestation observées dans la RBY présentent des différences importantes entre les périodes et les zones étudiées (concession de l'INERA, villages, axes routiers et cours d'eau). Avant 2003, le taux mesuré de 0,18\% de déforestation nette annuelle est relativement faible. II reste cependant légèrement supérieur à celui de 0,13 \% par an mesuré dans la zone d'Isangi (Katembera Ciza et al., 2015) et inférieur au taux national au cours de la même période (0,20\% par an ; Duveiller et al., 2008). Ce taux annuel de déforestation a fortement augmenté depuis 2003, atteignant 0,38 \% par an. Au cours de la période 2003-2016, il est devenu supérieur à la moyenne nationale estimée à environ 0,22 \% par an (De Wasseige et al., 2012). Il reste toutefois relativement bas par rapport à d'autres régions du pays, comme dans le domaine de chasse et la réserve de Bombo-Lumene, situés non loin de Kinshasa (0,46\% par an entre 2000 et 2015 ; Muyaya Kalambay et al., 2016) ; et très bas par rapport à l'Amérique tropicale $(0,51 \%)$ ou à l'Asie tropicale (0,58\% ; Gillet et al., 2016).

Le taux de déforestation plus élevé au cours de la seconde période (tableau II) peut être mis en parallèle avec l'importante augmentation de la population humaine depuis le milieu des années 1980 (tableau V), qui s'est accélérée depuis le début des années 2000 (Bamba et al., 2010). Diverses études ont également mis en évidence une corrélation entre la modification du couvert forestier dans le bassin

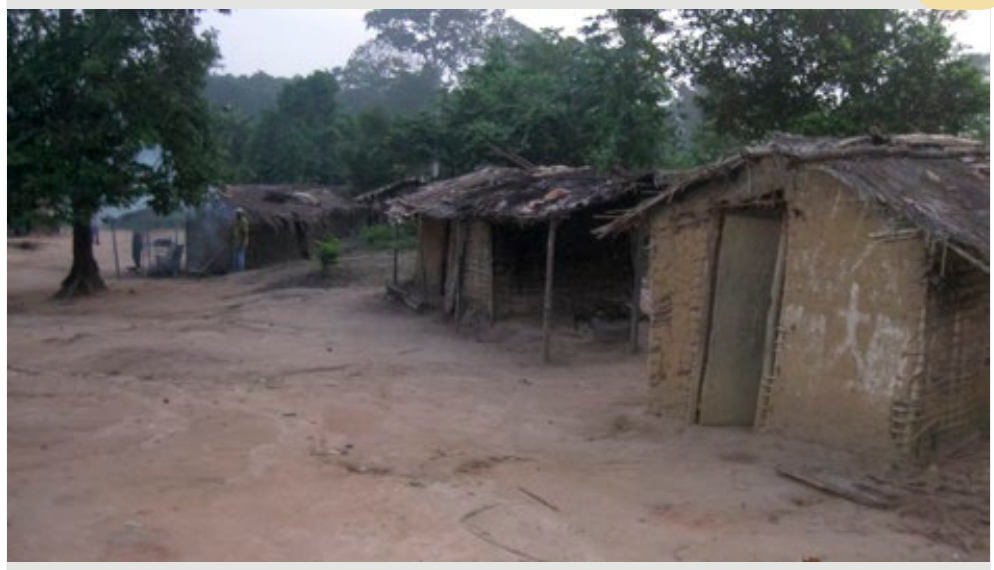

Photo 2.

Campement des exploitants d'or et de diamants.

Photo J. Kyale Koy.

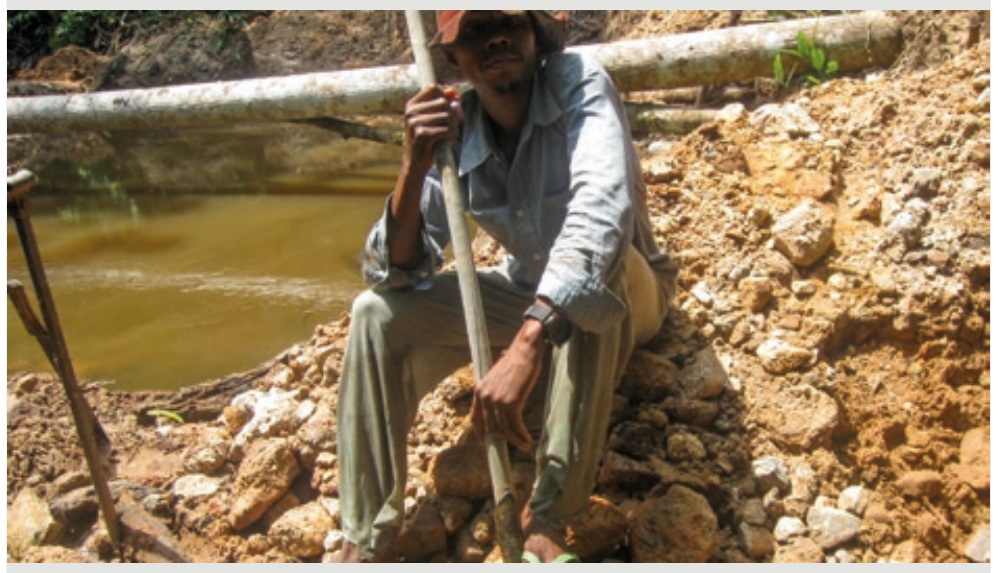

Photo 3.

Exploitation minière artisanale en amont de la rivière Lobilo. Photo J. Kyale Koy.

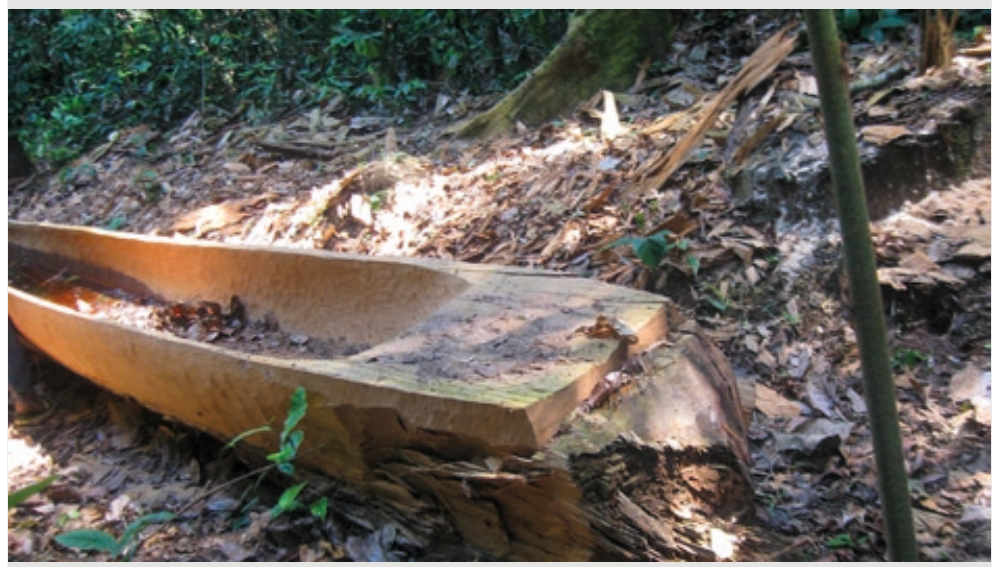

Photo 4.

Fabrication de pirogues par les résidents du campement Sembe.

Photo J. Kyale Koy. 
Tableau V.

Évolution démographique dans la région de la Réserve de biosphère de Yangambi.

\begin{tabular}{|c|c|c|c|c|c|}
\hline \multirow[t]{2}{*}{ Entités } & \multirow{2}{*}{$\begin{array}{c}\text { Superficie } \\
\left(\mathrm{km}^{2}\right)\end{array}$} & \multicolumn{2}{|c|}{1984} & \multicolumn{2}{|c|}{2015} \\
\hline & & Population & $\begin{array}{c}\text { Densité } \\
\left(\mathrm{hab} / \mathbf{k m}^{2}\right)\end{array}$ & Population & $\begin{array}{c}\text { Densité } \\
(\text { hab./km²) }\end{array}$ \\
\hline Yangambi & 840 & 21884 & 26,1 & 37679 & 44,9 \\
\hline Turumbu & 4600 & 25154 & 5,5 & 79098 & 17,2 \\
\hline Bengamisa & 5797 & 43605 & 7,5 & 110154 & 19 \\
\hline Total & 11237 & 90643 & 8,1 & 226931 & 20,2 \\
\hline
\end{tabular}

travailleurs ont été envahis par une population à la recherche de logement. Les grandes plantations créées pour la recherche agronomique et le fonctionnement de l'INERA, mal entretenues, furent largement abandonnées au profit des travailleurs et des paysans qui y ont installé leurs champs vivriers (Kombele, 2004). En outre, depuis les années 1950, la bourgade a acquis au cours du temps divers statuts administratifs qui ont favorisé l'installation de divers agents de l'État ainsi que celle de l'IFA (Institut facultaire des sciences agronomiques). Depuis 2013, Yangambi dispose d'ailleurs d'un statut de «ville » (décret $n^{\circ} 13 / 002$ de 13 juin 2013).

Ces facteurs permettent d'expliquer, dans une large mesure, qu'une grande partie de la « déforestation massive » qui est interve-

plupart des ménages ne disposent pas de source de revenus financiers du fait de la rareté de l'emploi privé ou de la modicité des salaires dans le secteur public, l'une des principales activités de survie est l'agriculture. En l'absence de systèmes culturaux intensifs, le recours général à l'agriculture itinérante sur brûlis, très consommatrice de terres et tributaire des forêts (Bamba et al., 2010), par les résidents de Yangambi-centre, des villages et des campements, explique les pertes du couvert végétal enregistrées. L'enquête a révélé qu'en moyenne, du fait du manque d'intrants ou de techniques adéquates, les agriculteurs défrichent environ 1 ha par an, en priorité dans les jachères et forêts secondaires, lorsqu'ils le peuvent, mais aussi dans les forêts primaires.

En outre, le contexte prévalant dans la région proche de Yangambi présente certaines particularités. La création de l'INERA en 1933, dénommé à l'époque INEAC, dans une zone très peu peuplée s'est rapidement accompagnée d'une importante concentration de main-d'œuvre destinée au fonctionnement de l'institution, avec plus de 4000 agents et leurs familles dès 1960 (Hiergens, 2010). En 1999, la construction de plusieurs camps pour les personnels qui ont pris leur retraite sur place a fixé cette population venant de diverses localités (Kombele, 2004). Au fur et à mesure de la diminution des activités de l'INERA, les camps des

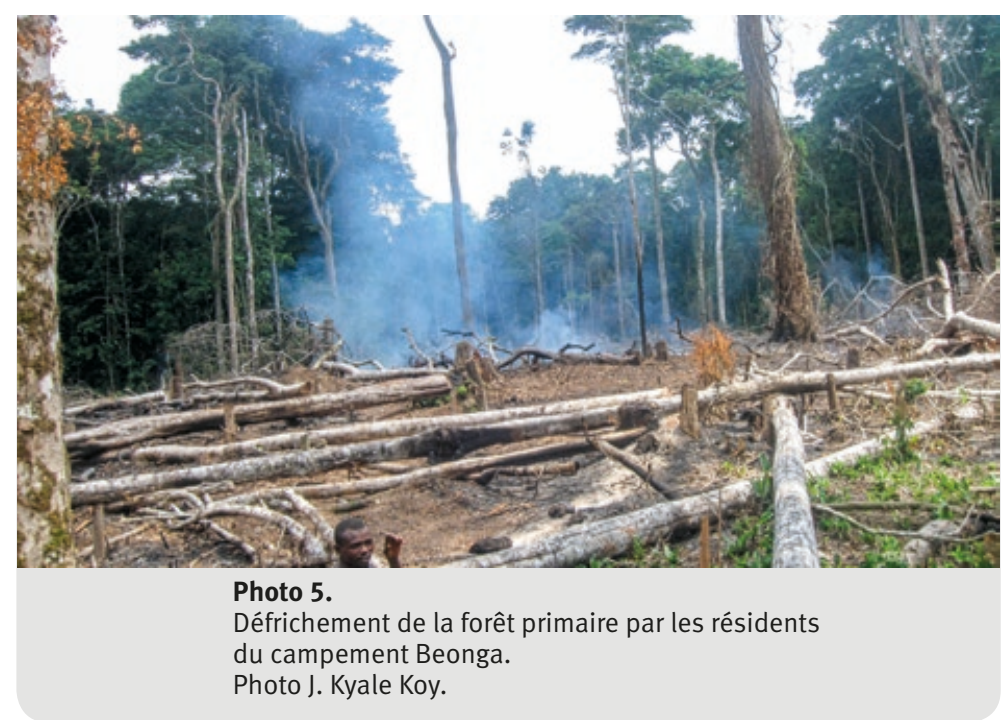

nue dans la RBY (par opposition à la « déforestation diffuse » du centre de la réserve) s'est exprimée essentiellement dans la concession de l'INERA, autour d'une station de recherche qui est devenue une importante bourgade. Dans un contexte d’État en faillite et de paupérisation, le statut du territoire géré par l'INERA a été un facteur de fixation de la population à Yangambi et d'aggravation de la dynamique de déforestation.

Dans la RBY, les deux guerres civiles qui ont déchiré le pays en 1995-1997 et 1998-2003 n'ont pas engendré une augmentation de la déforestation, comme cela a été relevé dans la réserve scientifique de Luo (Nackoney et al., 2014) et a été confirmé dans d'autres pays (Sayer et al., 2016). C'est plutôt après ces périodes de troubles que la déforestation s'est accrue fortement, en particulier aux dépens des forêts primaires (tableau II). Une relative stabilité sociale et une amélioration de certaines conditions de vie peuvent expliquer l'augmentation des taux de déforestation lors de la seconde période. En effet, la réhabilitation de la route Kisangani-Yangambi à partir de 2007 et les interventions des partenaires au développement, par des projets de renforcement des capacités institutionnelles, de relance de l'agriculture et de réhabilitation de la RBY (entre 2007 et 2016), peuvent avoir favorisé l'attractivité de la région de Yangambi et le développement agricole qui s'en est suivi. Le marché de Yangambi a pris de l'ampleur, attirant aussi par ricochet des migrants œuvrant dans divers secteurs (commerce, emplois formels et/ou informels).

D’autres grandes zones de déforestation massive se sont développées, d'une part, dans la région de Bengamisa, la plus proche du grand centre urbain de Kisangani et la mieux desservie par le réseau routier (à l'est), et, d'autre part, près du fleuve Congo vers Lilanda et le centre administratif d'Isangi (au sud-ouest). Dans ces deux cas, les facilités de transport et d'échanges, par route ou par fleuve, et la relative proximité à un grand centre de consommation semblent constituer les moteurs de déforestation. Cela a favorisé l'extension des complexes ruraux mêlant parcelles cultivées, jachères et forêts plus ou moins secondarisées (Potapov et al., 2012 ; Molinario et al., 2015). 
Toutefois, l'existence de réseaux routiers entourant ou pénétrant la réserve (figure 2) expose celle-ci aux impacts négatifs sur son couvert forestier. La construction et l'extension des réseaux routiers s'accompagnent souvent d'impacts négatifs sur le couvert forestier (Gillet et al., 2016). La RBY est exposée aux menaces d'anthropisation dues à son faible éloignement géographique aux axes routiers qui facilitent l'installation des campements et villages riverains.

Pour chacune des périodes étudiées, les forêts primaire et secondaire ont enregistré des taux de déforestation différents. Au cours de la première période (1986-2003), l'étude a révélé un taux de déforestation relativement élevé dans la forêt secondaire (1,8\%) par rapport à celui de la forêt primaire (1,2\% ; tableau II). Cela s'explique en particulier par le fait que le processus de spoliation des plantations de l'INERA a connu son intensification à cette période, suite à l'effondrement progressif de l'institution (Hiergens, 2010). Les forêts secondaires sont d'ailleurs très visibles le long des routes (reconstitution du couvert forestier après les anciens défrichements) et des cours d'eau qui arrosent la réserve (perturbation des écosystèmes par l'exploitation des feuilles de raphia et acheminement des pirogues vers le fleuve par les populations). De plus, la surexploitation des jachères dans des cycles culturaux de plus en plus courts à proximité de Yangambi, entraînant un appauvrissement des sols, incite les paysans à recourir à la forêt secondaire pour défricher de nouveaux champs. Comme un peu partout en Afrique subsaharienne, l'installation des champs dans la forêt secondaire offre aux paysans des avantages liés à l'adaptation des cultures très commercialisées (riz et maïs), à l'abattage facile des arbres, à la maximisation de l'espace utilisé pour les cultures et au faible envahissement des cultures par les herbes (De Wachter, 1997).

Au cours de la seconde période (2003-2016), les résultats montrent au contraire que la déforestation a été beaucoup plus forte dans la forêt primaire (4,5\%) que dans la forêt secondaire $(0,4 \%)$. Ce résultat est différent de celui obtenu par Katembera Ciza et al. (2015), montrant un taux élevé de déforestation dans la forêt secondaire de la région d'Isangi. Cette différence est due au fait que les ressources naturelles (mines, bois, gibiers, terres agricoles libres de toute appropriation) présentes dans la RBY amènent les riverains à s'installer de façon permanente dans des zones auparavant non défrichées. En y installant des campements, les populations rurales procèdent en particulier à l'ouverture des champs aux dépens de la forêt primaire (photo 5).

Au niveau des villages, le premier défricheur de la forêt primaire obtient le droit de l'usufruit (De Wachter, 1997). Par cette « mise en valeur » de la forêt (" droit de la hache "), il sécurise de nouvelles portions de forêts et se constitue une réserve d'espaces pour y développer des productions agricoles vivrières (autoconsommation) ou de rapport (destinées à être commercialisées). Dans les zones où la densité de population et les besoins en terres augmentent, ce facteur d'appropriation du sol (ou du moins de sécurisation coutumière des droits d'usage) est sans conteste une source majeure de déforestation.
Au cours de la première période (1986-2003), la pression sur les terres s'est surtout exercée à Yangambi, dans la concession de l'INERA, où la réserve en forêt secondaire était suffisante pour absorber cette demande en terres, du fait de l'attrait de la petite agglomération, de l'installation des camps de retraités et de la part active de l'INERA dans le démantèlement de son domaine. Au cours de la seconde période (2003-2016), la pression pour les terres a continué à Yangambi et a débordé partiellement des forêts secondaires et/ou des plantations. C'est surtout dans les parties est (vers Bengamisa) et sud de la réserve (vers Lilanda) et de Yakako à Bosukulu que l'augmentation du défrichement s'est manifestée, dans des zones couvertes jadis par la forêt primaire. L'augmentation de la population rurale, l'existence des marchés ruraux hebdomadaires ou bihebdomadaires et la demande de produits agricoles par la population de Kisangani, estimée à environ 1600000 habitants (soit une densité de plus de $800 \mathrm{hab} . / \mathrm{km}^{2}$ ), poussent les ruraux à augmenter leurs surfaces agricoles aux dépens de la forêt primaire de ces zones (Bamba et al., 2010).

Comme cela a été souligné dans d'autres études (Ernst et al., 2012 ; Potapov et al., 2012), les enquêtes ont révélé que l'exploitation des minerais s'est fortement accrue dans la réserve, favorisant la destruction et la dégradation de la forêt primaire selon une dynamique de déforestation diffuse de petites zones déforestées éparpillées dans la matrice forestière. Celle-ci s'exprime en particulier le long des cours d'eau, mais aussi entre ceux-ci. La plupart des sites miniers actifs dans le passé sont maintenant épuisés et convertis en campements de chasseurs et d'artisans fabricants de pirogues (figure 2). Les campements actuellement actifs comprennent entre 3 et 60 résidents permanents, avec une moyenne de 12 et un écart-type de 2,1. Ces campements occupent des surfaces allant de un à plus d'une dizaine d'hectares. Au-delà de l'augmentation des superficies des forêts secondaires, des jachères et des sols nus, l'impact des activités des résidents des campements sur les peuplements fauniques et sur certains végétaux fournissant des produits forestiers non ligneux n'est pas négligeable, comme cela a par exemple été montré pour la chasse (Nackoney et al., 2014).

Par ailleurs, la forêt primaire, peu ou pas dégradée, occupe encore la plus grande superficie des forêts de la RBY (environ $86 \%$ du territoire ; tableau II) : sa valeur botanique et écosystémique reste relativement bonne, comparée à sa valeur faunique, qui a été plus fortement dégradée, s’agissant en particulier des mammifères (Toirambe et al., 2011). Les techniques culturales et les instruments de travail agricole étant rudimentaires, les paysans défrichent difficilement de vastes étendues dans ce type d'écosystèmes forestiers (De Wachter, 1997). En outre, cultiver très loin du village rend plus pénible l'activité des paysans et paysannes. C'est pour cela que les champs vivriers responsables de la déforestation sont localisés le long des routes, autour des zones habitées ou à l'extrême est et sud de la réserve, non loin des axes routiers ou du fleuve.

Enfin, le coefficient Kappa obtenu pour toutes les dates étant supérieur à $95 \%$, les cartes d'occupation des sols présentées semblent être de très bonne qualité suivant l'échelle de Landis et Koch (1977). Cela consolide le soubassement de l'analyse de transition réalisée entre les différentes dates. 


\section{Conclusion}

La présente analyse de la déforestation dans la Réserve de biosphère de Yangambi (RBY) a permis d'affiner la perception des dynamiques d'occupation des sols et de la déforestation en RDC. La combinaison d'outils de télédétection, de vérité terrain et d'enquêtes a permis de vérifier si la dynamique de déforestation est variable dans les différentes zones de la réserve et au cours du temps. Deux périodes, 1986-2003 et 2003-2016, ont constitué la base de comparaison des données spatiales en vue de cerner les taux de déforestation et d'identifier les zones fortement anthropisées. La seconde période a connu un taux annuel de déforestation élevé (0,38\%, soit une perte de 973 ha/an), comparativement à la première $(0,18 \%$, soit 479 ha/an), du fait d'une plus grande stabilité de la zone depuis la fin des guerres civiles, dans un contexte d'État en faillite et d'une augmentation de la demande urbaine, notamment de la ville de Kisangani.

Comme cela a été montré dans d'autres travaux (Wardell et al., 2003 ; Bamba et al., 2010 ; Ernst et al., 2012 ; Potapov et al., 2012), l'agriculture itinérante sur brûlis (en l'absence d'une agriculture commerciale industrielle), l'exploitation forestière et l'exploitation minière constituent les facteurs directs de déforestation les plus décisifs. D’autres facteurs qui agissent plutôt sur la structure des forêts et en favorisent la dégradation peuvent aussi être invoqués, tels que la construction de pirogues, voire la collecte de produits forestiers non ligneux telles les feuilles du palmier raphia. La pression démographique, non accompagnée de l'amélioration du niveau de vie des communautés locales ou de celle des techniques agricoles, et le développement du réseau routier (favorisant les échanges commerciaux) constituent des facteurs sous-jacents importants. L'ampleur que prennent ces facteurs d'une zone à une autre entraîne une répartition inégale de la perte du couvert forestier dans la RBY. Sur l'ensemble du territoire et durant la période concernée par l'étude, on peut identifier quatre situations de changement d'occupation des sols :

- la concession de l'INERA, qui s'est transformée au fil du temps en une véritable bourgade, où la déforestation a été la plus active depuis le milieu des années 1980, avec une accélération depuis le milieu des années 2000 ;

- les zones proches d'axes de circulation majeurs (routes carrossables, fleuve), qui étaient encore largement forestières au milieu des années 2000 , mais qui présentent une déforestation massive depuis cette époque, la forêt étant remplacée par des champs, des sols nus et des jachères ; - les zones situées sur des axes routiers plus ou moins abandonnés où la déforestation avait déjà débuté autour des villages avant le milieu des années 2000 et s'est exacerbée depuis cette époque, mais sans atteindre pour le moment les niveaux de déforestation de masse des deux zones précédentes ;

- enfin, les zones isolées du cœur de la RBY, loin des principaux réseaux de communication, uniquement accessibles par des petites rivières et des sentiers, où la déforestation diffuse a débuté avant les années 2000 et s'est étendue depuis lors sur l'ensemble du territoire de la réserve.
Par ailleurs, même si les forêts primaires recouvrent encore plus de $86 \%$ de la réserve, les nombreuses activités humaines et les dynamiques socio-économiques à l'œuvre mettent en péril ce patrimoine naturel inclus dans le réseau mondial des réserves de biosphère. Le plan d'aménagement proposé depuis plusieurs années (Toirambe et al., 2011) n'étant pas mis en œuvre, l'identification de ces différentes dynamiques sociales et environnementales sur le territoire de la RBY devrait permettre de l'affiner et de proposer des règles de gestion et de gouvernance mieux adaptées aux dynamiques en cours.

\section{Remerciements}

Les auteurs témoignent de leur gratitude à l'Union européenne (GCCA) pour les fonds alloués à la recherche en République démocratique du Congo via le projet Forêts et changement climatique au Congo (FCCC). Ils remercient le Centre pour la recherche forestière internationale (CIFOR), le Centre de coopération internationale en recherche agronomique pour le développement (CIRAD) et l'Université de Kisangani (UNIKIS) pour l'encadrement scientifique des recherches doctorales du premier auteur. Le bureau d'études Resources and Synergies Development (RSD) est remercié pour l'efficacité de sa gestion logistique durant la période de collecte des données.

\section{Références bibliographiques}

Arabi B., Salama S. Mhd, Wernand M. R., Verhoef W., 2016. MOD2SEA: A coupled atmosphere-hydro-optical model for retrieval of chlorophyll-a from remote sensing observations in complex turbid waters. Remote Sensing, 722 (8): 1-22. https://www.mdpi.com/2072-4292/8/9/722/htm

Bamba I., Barima Y. S. S., Bogaert J., 2010. Influence de la densité de la population sur la structure spatiale d'un paysage forestier dans le bassin du Congo en R. D. Congo. Tropical Conservation Science, 3 (1) : 31-44. https:// tropicalconservationscience.mongabay.com/content/ v3/10-03-29 31-44 bamba et al.pdf

Barima Y. S. S., Barbier N., Bamba I., Traore D., Lejoly J., Bogaert J., 2009. Dynamique paysagère en milieu de transition forêt-savane ivoirienne. Bois et Forêts des Tropiques, 299 (1) : 15-25. https://doi.org/10.19182/bft2009.299. a20419

Beguin H., 1958. Géographie humaine de la région de Bengamisa. Bruxelles, Belgique, Publication de l'INEAC, série scientifique, 74, 69 p.

Caloz R., Collet C., 2001. Précis de télédétection. Traitements numériques d'images de télédétection. Sainte-Foy, Canada, Presses de l'Université du Québec et Agence universitaire de la francophonie, $386 \mathrm{p}$.

De Wachter P., 1997. Économie et impact de l'agriculture itinérante Badjoué (sud-Cameroun). Civilisations, 44 : 62-93. https://journals.openedition.org/civilisations/1611 
De Wasseige C., de Marcken P., Bayol N., Hiol Hiol F., Mayaux P., Desclée B., et al., 2012. Les forêts du bassin du Congo État des forêts 2010. Luxembourg, Office des publications de l'Union européenne, $276 \mathrm{p}$.

Donis C., 1956. La forêt dense congolaise et l'état actuel de sa sylviculture. Bulletin Agricole du Congo Belge, 2 (47) : 261-303.

Duveiller G., Defourny P., Desclée B., Mayaux P., 2008. Deforestation in Central Africa: Estimates at regional, national and landscape levels by advanced processing of systematically-distributed Landsat extracts. Remote Sensing of Environment, 112: 1969-1981. https://doi.org/10.1016/j. rse.2007.07.026

Ernst C., Verhegghen A., Mayaux P., Hansen M., Defourny P., 2012. Cartographie du couvert forestier et des changements du couvert forestier en Afrique centrale. In : De Wasseige C., De Marcken P., Bayol N., Hiol Hiol F., Mayaux P., Desclée B., et al. (éds). Les forêts du bassin du Congo - État des forêts 2010, Luxembourg, Office des publications de l'Union européenne, 23-42. https://www.observatoire-comifac.net/ docs/edf2010/FR/Etat-des-forets 2010.pdf

Gillet P., Vermeulen C., Feintrenie L., Dessard H., Garcia C., 2016. Quelles sont les causes de la déforestation dans le bassin du Congo ? Synthèse bibliographique et études de cas. BASE, 20 (2) : 183-194. http://www.pressesagro.be/ base/text/v20n2/183.pdf

Gosse J.-P., 1963. Le milieu aquatique et l'écologie des poissons dans la région de Yangambi. Annales du MRAC, Sciences zoologiques, 116 (8) : 115- 270.

Hiergens T., 2010. Un centre de recherche précieux enfoui sous des tonnes de poussière. À Yangambi subsiste l'espoir que tout redémarre un jour. Journal de la Coopération Belge, 3 : 19-20. https://rdcongo.diplomatie.belgium.be/sites/ default/files/content/50 ans rdc.pdf

INEAC, 1939. Rapport annuel pour l'exercice 1939. Hors série 1940. (Consulté : Archives de l'INERA, Yangambi.)

INS, 1984. Recensement scientifique de la population, Haut-Zaïre, Zones, Collectivités, Cités, Groupements, Quartiers. Kinshasa, RDC, Ministère du Plan, 162 p.

Katembera Ciza S., Mikwa J. F., Cirhuza Malekezi A., Gond V., Boyemba Bosela F., 2015. Identification des moteurs de déforestation dans la région d'Isangi, République démocratique du Congo. Bois et Forêts des Tropiques, 324 (2) : 29-38. Doi : 10.19182/bft2015.324.a31264

Kombele B. M., 2004. Diagnostic de la fertilité des sols dans la cuvette centrale congolaise. Cas des séries Yangambi et Yakonde. Thèse en sciences agronomiques et ingénierie biologique, Faculté universitaire des sciences agronomiques de Gembloux, Belgique, 456 p.

Landis J. T., Koch G. G., 1977. The Measurement of Observer Agreement for Categorical Data. Biometrics, 33: 159-174. https://www.dentalage.co.uk/wp-content/uploads/2014/09/ landis jr koch gg 1977 kappa and observer agreement. pdf
Latendresse C., Jobin B., Maisonneuve C., Sebbane A., Grenier M., 2008. Changements de l'occupation du sol dans le Québec Méridional entre 1993 et 2001. Le Naturaliste Canadien, 132 (1): 14-23. https://provancher-my.sharepoint. com/personal/accesdocuments provancher org/Documents/Documents/Num\%C3\%A9ros\%20Naturaliste\%20 Canadien/Haute Res/Naturaliste Canadien 132-1 Hiver 2008.pdf

Mas J. F., 2000. Une revue des méthodes et des techniques de télédétection du changement. Canadian Journal of Remote Sensing, 26 (4) : 349-362. https://doi.org/10.108 0/07038992.2000.10874785

Mohymont B., Demarée G. R., 2006. Courbes intensité-durée-fréquence des précipitations à Yangambi, Congo, au moyen de différents modèles de type Montana. Hydrological Sciences, Journal des Sciences Hydrologiques, 51 (2) : 239-253. https://www.tandfonline.com/doi/pdf/10.1623/ hysj.51.2.239? needAccess=true

Molinario G., Hansen M. C., Potapov P. V., 2015. Forest cover dynamics of shifting cultivation in the Democratic Republic of Congo: a remote sensing-based assessment for 20002010. Environmental Research Letters, 10 (9). http://iopscience.iop.org/article/10.1088/1748-9326/10/9/094009/ $\underline{\mathrm{pdf}}$

Muyaya Kalambay B., Rudant J. P., Lumbuenamo R., Beland M., Riera B., 2016. Dynamique spatiale du domaine de chasse et réserve de Bombo Lumene entre 2000 et 2015 par imagerie satellitaire optique. International Journal of Innovation and Applied Studies, 2 (18) : 559-568. http://www. ijias.issr-journals.org/abstract.php?article=|J|AS-16-184-01

Nackoney J., Molinario G., Potapov P., Turubanova S., Hansen M. C., Furuichi T., 2014. Impacts of civil conflict on primary forest habitat in northern Democratic Republic of the Congo, 1990-2010. Biological Conservation, 170: 321-328. https://langint.pri.kyoto-u.ac.jp/ai/intra data/JanetNackoney/NackoneyJ2014-BC.pdf

Oszwald J., Gond V., Dolédec S., Lavelle P., 2011. Identification d'indicateurs de changement d'occupation du sol pour le suivi des mosaïques paysagères. Bois et Forêts des Tropiques, 307 1) : 7-21. Doi : 10.19182/bft2011.307.a20484

Pélissier C., De Marcken P., Mapilangawa Tsaramu J.-J., Wilungula Balongelwa C., 2015. République démocratique du Congo. In : Doumenge C., Palla F., Scholte P., Hiol Hiol F., Larzillière A. (éds). Aires protégées d'Afrique centrale État 2015. Kinshasa, République démocratique du Congo et Yaoundé, Cameroun, OFAC, 111-147. https://www. observatoire-comifac.net/docs/edAP2015/FR/EdAP 2015 RDCongo.pdf

Poll M., Gosse J.-P., 1963. Contribution à l'étude systématique de la faune ichthyologique du Congo central. Annales du MRAC, 116 (8) : 45-114.

Potapov P. V., Turubanova S. V., Hansen M. C., Adusei B., Broich M., Altstatt A., et al., 2012. Quantifying forest cover loss in Democratic Republic of the Congo, 2000-2010, with Landsat ETM+ data. Remote Sensing of Environment, 122: 106-116. https://doi.org/10.1016/i.rse.2011.08.027 
Rosas J., Houborg R., Mc Cabe M. F., 2017. Sensivity of Landsat 8 surface temperature estimates to atmospheric profile data: a study using MODTRAN in Dryland irrigated systems. Remote Sensing, 988 (9): 1-27. https://www.mdpi. com/2072-4292/9/10/988/htm

Sayer J., Endamana D., Boedhihartono A. K., Ruiz-Perez M., Breuer T., 2016. Learning from change in the Sangha Tri-National landscape. International Forestry Review, 18 (S1): 130-139. https://doi.org/10.1505/146554816819683771

Secteur Bamanga, 2015. Rapport annuel, exercice 2015. Tableau synoptique de recensement de la population congolaise. (Consulté : service d'état civil du secteur Bamanga à Bengamisa.)

Secteur Turumbu, 2015. Rapport de premier trimestre, exercice 2015. Tableau synoptique de recensement de la population congolaise. (Consulté : service d'état civil du secteur Turumbu à Yaselia.)

Semeki Ngabinzeke J., Linchant J., Quevauvillers S., Kahindo Muhongya J.-M., Lejeune P., Vermeulen C., 2016, Cartographie de la dynamique de terroirs villageois à l'aide d'un drone dans les aires protégées de la République démocratique du Congo. Bois et Forêts des Tropiques, 330 (4) : 69-83. http://bft.cirad.fr/cd/BFT 330 69-83.pdf

Soungalo S., Kadeba A. I., Nacoulma B. M. I., Traoré S., Bachmann Y., Thiombiano A., 2015. Impact des activités anthropiques sur la dynamique de la végétation de la réserve partielle de faune de Pama et de ses périphéries (sud-est du Burkina Faso) dans un contexte de variabilité climatique. Journal of Applied Biosciences, 87 : 8047-8064. http://m. elewa.org/Journals/wp-content/uploads/2015/03/5.-soulama-Abstract-Vol.-87.pdf

Thi-Thanh-Hiên Pham, Dong-Chen He, 2013. Classification orientée objet d'une image quickbird de la ville de Hanoi (Viêtnam) : étude empirique des paramètres de segmentation. Télédétection, Éditions scientifiques GB, 11 (2) : 287-305. https://halshs.archives-ouvertes.fr/ halshs-01134611/document

Toirambe B., Adebu B., Nsenga L., 2011. Plan d'aménagement de la réserve de biosphère de Yangambi. Kinshasa, RDC, WWF, $77 \mathrm{p}$.

Van Wambeke A., Evrard C., 1954. Carte du sol et de la végétation du Congo belge et du Ruanda-Urundi. 6. Yangambi, planchette 1 : Weko A et B. Notice explicative de la carte des sols et de la végétation. Bruxelles, Belgique, publication de I'INEAC, $23 \mathrm{p}$.

Ville de Yangambi, 2015. Rapport annuel, exercice 2015. Tableau synoptique de recensement de la population, statistique par entité. (Consulté : service d'état civil de la ville de Yangambi.)

Wafo Tabopda G., Fotsing J. M., 2010. Quantification de l'évolution du couvert végétal dans la réserve forestière de Laf-Madjam au nord du Cameroun par télédétection satellitaire. Sécheresse, 21 (3): 169-78.
Wardell D. A., Reenberg A., Tøttrup C., 2003. Historical footprints in contemporary land use systems: forest cover changes in savannah woodlands in the Sudano-Sahelian zone. Global Environmental Change, 13: 235-254. http:// lasyre.dk/secure/litterature/Wardell 2003.pdf

Walters G., Ngagnia Ndjabounda E., Ikabanga D., Biteau J. P., Hymas O., White L. J.T., etal., 2016. Peri-urban conservation in the Mondah forest of Libreville, Gabon: Red List assessments of endemic plant species, and avoiding protected area downsizing. Oryx, 50 (3): 419-430. https://www.cambridge.org/ core/journals/oryx/article/periurban-conservation-in-themondah-forest-of-libreville-gabon-red-list-assessmentsof-endemic-plant-species-and-avoiding-protected-areadownsizing/2F9535A82831E7845D33738CADE33364/ core-reader

Willis K. S., 2015. Remote sensing change detection for ecological monitoring in United States protected areas. Biological Conservation, 182: 233-242. https://doi.org/10.1016/j. biocon.2014.12.006

Zhang Q., Justice C. O., Jiang M., Brunner J., Wilkie D., 2006. A GIS-based assessment on the vulnerability and future extent of the tropical forests of the Congo Basin. Environmental Monitoring and Assessment, 114: 107-121. https:// link.springer.com/article/10.1007/s10661-006-2015-3

\section{Kyale Koy et al. - Contribution des auteurs}

\begin{tabular}{|c|c|}
\hline Rôle du contributeur & Noms des auteurs \\
\hline Conceptualisation & $\begin{array}{l}\text { J. Kyale Koy, D. A. Wardell, } \\
\text { A. M. Monga Ngonga }\end{array}$ \\
\hline Gestion des données & $\begin{array}{l}\text { J. Kyale Koy, J.-F. Mikwa, } \\
\text { J. M. Kabuanga }\end{array}$ \\
\hline Analyse formelle & J.-F. Mikwa \\
\hline $\begin{array}{l}\text { Acquisition } \\
\text { du financement }\end{array}$ & J. Kyale Koy \\
\hline $\begin{array}{l}\text { Enquête } \\
\text { et investigation }\end{array}$ & J. Kyale Koy \\
\hline Méthodologie & J. Oszwald \\
\hline Supervision & D. A. Wardell, A. M. Monga Ngonga \\
\hline Visualisation & J. M. Kabuanga \\
\hline $\begin{array}{l}\text { Écriture - Préparation } \\
\text { de l’ébauche originale }\end{array}$ & $\begin{array}{l}\text { J. Kyale Koy, C. Doumenge, } \\
\text { D. A. Wardell, A. M. Monga Ngonga }\end{array}$ \\
\hline $\begin{array}{l}\text { Écriture - Révision } \\
\text { et édition }\end{array}$ & $\begin{array}{l}\text { J. Kyale Koy, D. A. Wardell, } \\
\text { J. M. Kabuanga, A. M. Monga Ngonga, } \\
\text { C. Doumenge }\end{array}$ \\
\hline
\end{tabular}

Bois et Forêts des Tropiques - Revue scientifique du Cirad

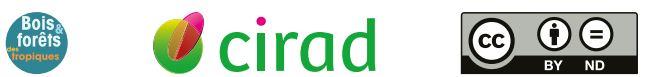

Cirad - Campus international de Baillarguet, 34398 Montpellier Cedex 5, France - Contact : bft@cirad.fr - ISSN : L-0006-579X 